\title{
Seroprevalence and risk factors of Brucella ovis in domestic sheep in Wyoming, USA
}

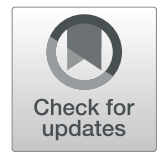

\author{
Molly Elderbrook', Brant Schumaker', Todd Cornish', Dannele Peck ${ }^{2}$ and Kerry Sondgeroth ${ }^{1 *}$ (D)
}

\begin{abstract}
Background: Brucella ovis causes a sexually transmitted, infectious disease of domestic sheep characterized by genital lesions and epididymitis in rams, placentitis and rare abortions in ewes, and neonatal mortality in lambs. This study was designed to 1) estimate animal and flock seroprevalence of B. ovis in sheep across Wyoming, USA, and 2) describe epidemiologic risk factors associated with seropositive sheep and flocks. For the animal seroprevalence estimate, 2423 blood samples were collected from sheep on 18 producer-selected operations and a questionnaire about possible risk factors was distributed. For the flock seroprevalence estimate, blood samples from 82 operations were obtained, including samples from the previous 18 operations and 64 additional operations that sent samples to the Wyoming State Veterinary Laboratory for diagnostic testing. Categorical risk factors were created based on questionnaires and submission forms. Sera was analyzed using the B. ovis enzyme-linked immunosorbent assay.

Results: Estimated true animal and flock seroprevalence were $0.53 \%$ (95\% Cl: $0.21-1.01 \% ; 22 / 2,423)$ and 22.5\% (95\% Cl: 14-32\%; 18/82), respectively. Using Fisher's exact and Mid-p exact tests to compare apparent seroprevalence with respect to possible risk factors, increased age and breed type were risk factors associated with seropositive sheep, while region and large flock size were risk factors associated with seropositive flocks.

Conclusions: Results from this study suggest few sheep have been exposed to B. ovis, but many flocks contain at least one seropositive animal. Each region in Wyoming contained at least one seropositive animal and flock, emphasizing the importance of disease-free documentation before purchasing new sheep. Aged sheep ( $\geq 6$ years of age) had the highest seroprevalence among age groups; hence, we propose the separation of young rams from older rams to help reduce disease spread outside the breeding season. Wool breeds (Rambouillet and Merino) may be less susceptible to B. ovis infection given they had the lowest animal seroprevalence of the breed types, and large flocks (> 100 breeding rams) had the highest seroprevalence of the flock size categories, likely due to more intensive management strategies that can contribute to the introduction and persistence of B. ovis infection in sheep and flocks.
\end{abstract}

Keywords: Brucella ovis, Sheep, Ram epididymitis, Seroprevalence, Wyoming

\section{Background}

Brucella ovis (B. ovis) is the causative agent of a sexually transmitted, infectious disease resulting in clinical or subclinical chronic disease in domestic sheep [1]. The disease was first described in Australia and New Zealand in 1953 $[2,3]$ and was later discovered in the United States [4]; however, disease likely occurs in most major sheepproducing countries [1]. During natural infection, disease is introduced into a flock after the reintroduction of an infected ram, and the most common route of disease spread

\footnotetext{
* Correspondence: ksondger@uwyo.edu

${ }^{1}$ Department of Veterinary Sciences, University of Wyoming, 1174 Snowy

Range Road, Laramie, WY 82070, USA

Full list of author information is available at the end of the article
}

is indirect ram-to-ram transmission via passive venereal contact with ewes during the breeding season [5-8]. Rams excrete $B$. ovis in semen intermittently for two or more years, and ewes occasionally excrete $B$. ovis in vaginal discharge and in milk near parturition; however, infection rarely persists for successive breeding seasons $[7,8]$. Disease transmission also occurs directly from ram-to-ram via oral and mucosal routes outside the breeding season $[7,9]$.

The most common clinical sign of B. ovis infection is epididymitis in rams, which can be detected on a breeding soundness examination (BSE) prior to the breeding season. A proper BSE consists of a physical exam to assess body condition, scrotal circumference, presence of ulcerative posthitis, or epididymitis, and a semen

(c) The Author(s). 2019 Open Access This article is distributed under the terms of the Creative Commons Attribution 4.0 International License (http://creativecommons.org/licenses/by/4.0/), which permits unrestricted use, distribution, and 
evaluation by microscopy to assess spermatozoa motility, morphology, and white blood cell count [10]. However, less than $50 \%$ of rams infected with $B$. ovis develop a palpable epididymitis, and infection with other bacterial agents can cause abnormalities in spermatozoa [10]. Therefore, diagnosis with the indirect enzyme-linked immunosorbent assay (ELISA) is preferred. The B. melitensis Rev. 1 vaccine is a live strain vaccine that can stimulate immunity against $B$. ovis [1]; however, disadvantages of vaccine use include the development of $B$. melitensis and B. ovis antibodies creating interference on serological tests, and human disease caused by accidental self-inoculation [11]. For these reasons, the B. melitensis Rev. 1 vaccine is prohibited from use in countries affected by $B$. ovis, but free from $B$. melitensis, including the U.S. $[1,5,12]$. Antibiotic treatment is often not financially feasible; hence, a test-and-slaughter management strategy is currently the most cost-effective option for eliminating B. ovis in flocks in the U.S. [5].

The economic repercussions of $B$. ovis include decreased fertility in rams, lower conception rates in ewes, and a reduction in the total number of healthy lambs born. Brucella ovis is a concern for many U.S. sheep operations, as lamb production accounts for a high percentage of gross annual sales [13]. Furthermore, indirect reproductive and financial costs associated with $B$. ovis include the purchase of additional rams to effectively breed ewes, culling of genetically valuable rams due to infection, repetitive serological testing to control disease, and prolonged lambing seasons that can be detrimental in states with severe weather.

Research regarding the importance of $B$. ovis in domestic sheep has been done primarily on rams in major sheep-producing regions $[9,14-20]$, but none have estimated seroprevalence or assessed possible risk factors associated with B. ovis in both rams and ewes in North American flocks. The information from this study will help fill the knowledge gap regarding seroprevalence of this reproductive disease in the U.S., aid in future management decisions for producers, and give insight into the relevance and epidemiology of $B$. ovis in ewes. The objective of this study was to estimate true animal and flock seroprevalence of $B$. ovis in study populations in Wyoming, and describe epidemiologic risk factors associated with seropositive sheep and flocks.

\section{Results}

\section{Animal seroprevalence and risk factors}

Among all sheep tested for B. ovis antibodies, 22 were seropositive, resulting in an apparent seroprevalence of $0.91 \%$ (95\% CI: $0.60-1.37 \%$ ) and an estimated true seroprevalence of $0.53 \%$ (95\% CI: $0.21-1.01 \%)$ in the study population when considering test sensitivity and specificity. Apparent animal seroprevalence and the odds of B. ovis exposure for potential risk factors are presented in Table 1.

Of the 2423 sheep sampled for this study, 12\% (298/ 2423) were from the Northwest region, $11 \%(255 / 2423)$ were from the Northeast region, 34\% (832/2423) were from the West region, $24 \%(572 / 2423)$ were from the South-central region, and 19\% (466/2423) were from the Southeast region. Sheep in the Northwest region had the highest apparent seroprevalence $(1.68 \%)$, but there was not a statistically significant difference between seroprevalence and the five regions $(p=0.113)$ according to the Fisher's exact test.

Rams comprised $61 \%$ of sheep sampled in this study (1477/2423) and ewes comprised 39\% (946/2423). Rams had a slightly higher apparent seroprevalence compared to ewes ( 0.95 and $0.85 \%$, respectively); however, this difference was not statistically significant $(p=0.812)$ according to the Mid-p exact test.

Of the 2242 sheep sampled with known ages, 7\% (147/ 2242) were lambs, $24 \%$ (538/2242) were yearlings, $59 \%$ (1337/2242) were adult sheep, and 10\% (220/2242) were aged sheep. No lambs were seropositive in this study; therefore, we eliminated this category from further analysis. Apparent seroprevalence was $0.56 \%$ in yearling sheep, $0.60 \%$ in adult sheep, and $2.27 \%$ in aged sheep, with a statistically significant difference between seroprevalence among these age categories $(p=0.044)$ (Table 1$)$ according to the Fisher's exact test. Furthermore, the odds of $B$. ovis exposure in aged sheep was about 4 times that of yearling sheep.

Of the 2375 sheep sampled with known breeds, $46 \%$ (1083/2375) were wool breeds, 37\% (890/2375) were multipurpose breeds, and 17\% (402/2375) were meat breeds. There was a statistically significant difference in seroprevalence between primary breed types, as $0.28 \%$ of wool breeds, $1.24 \%$ of meat breeds, and $1.35 \%$ of multi-purpose breeds were seropositive $(p=0.012)$ (Table 1$)$ according to the Fisher's exact test. The odds of $B$. ovis exposure in multi-purpose and meat breeds was 4.7 and 4.4 times, respectively, the odds of $B$. ovis exposure in wool breeds.

Of the 2423 samples collected in this study, 69\% $(1669 / 2423)$ were collected before the breeding season, and 31\% (754/2423) were collected after the breeding season. Apparent animal seroprevalence of sheep sampled before the breeding season was $1.08 \%$ compared to $0.53 \%$ for sheep sampled after the breeding season, and this difference was not statistically significant $(p=0.191)$ according to the Mid-p exact test.

Two variables (age category and primary breed type) were incorporated into a multiple logistic regression model. The wool breed type and aged sheep categories had $p$-values of 0.035 and 0.036 , respectively. The negative coefficient for the wool breed type and positive coefficient for the aged sheep category suggests that, if all other variables being equal, the wool breeds are less 
Table 1 Apparent animal seroprevalence and odds of B. ovis exposure for risk factors in Wyoming, USA

\begin{tabular}{|c|c|c|c|c|}
\hline Risk Factor & $\mathrm{N}$ & Seroprevalence $^{a}$ & Odds Ratio $(95 \% \mathrm{Cl})$ & $P$ value \\
\hline \multicolumn{5}{|l|}{ Region } \\
\hline Northeast & 298 & $0.39 \%(1 / 255)$ & Reference & - \\
\hline Northwest & 255 & $1.68 \%(5 / 298)$ & $3.90(0.59$ to 103.51$)$ & 0.346 \\
\hline West & 832 & $0.48 \%(4 / 832)$ & 1.11 (0.15 to 30.48 ) & 0.927 \\
\hline South-central & 572 & $1.57 \%(9 / 572)$ & 3.60 (0.66 to 90.16$)$ & 0.346 \\
\hline Southeast & 466 & $0.64 \%(3 / 466)$ & 1.51 (0.17 to 43.49) & 0.927 \\
\hline \multicolumn{5}{|l|}{ Gender } \\
\hline Rams & 1477 & $0.95 \%(14 / 1477)$ & Reference & - \\
\hline Ewes & 946 & $0.85 \%(8 / 946)$ & 0.90 (0.35 to 2.13) & 0.812 \\
\hline \multicolumn{5}{|l|}{ Age Groups ${ }^{b}$} \\
\hline Lambs & 147 & $0.00 \%(0 / 147)$ & - & - \\
\hline Yearlings & 538 & $0.56 \%(3 / 538)$ & Reference & - \\
\hline Adults & 1337 & $0.60 \%(8 / 1337)$ & 1.04 (0.29 to 5.02$)$ & 0.955 \\
\hline Aged & 220 & $2.27 \%(5 / 220)$ & 4.06 (0.95 to 21.20$)$ & 0.118 \\
\hline \multicolumn{5}{|l|}{ Primary Breed Type ${ }^{b}$} \\
\hline Wool & 1083 & $0.28 \%(3 / 1083)$ & Reference & - \\
\hline Meat & 402 & $1.24 \%(5 / 402)$ & 4.44 (1.04 to 23.12$)$ & 0.044 \\
\hline Multi-purpose & 890 & $1.35 \%(12 / 890)$ & 4.73 (1.48 to 21.79$)$ & 0.014 \\
\hline \multicolumn{5}{|c|}{ Time of Sample Collection } \\
\hline After Breeding & 754 & $0.53 \%(4 / 754)$ & Reference & - \\
\hline Before Breeding & 1669 & $1.08 \%(18 / 1669)$ & 1.98 (0.73 to 7.06$)$ & 0.191 \\
\hline
\end{tabular}

a Parenthesis indicate proportion of seropositive sheep per category

${ }^{b}$ Indicates significant difference between seroprevalence by Fisher's exact test at $p<0.05$

likely to be seropositive and aged sheep are more likely to be seropositive when tested for B. ovis antibodies.

\section{Flock seroprevalence and risk factors}

Among all flocks tested for B. ovis antibodies, 18 contained at least one seropositive animal, resulting in an apparent seroprevalece of $22.0 \%$ (95\% CI: 14.4-32.1\%) and an estimated true seroprevalence of $22.5 \%$ (95\% CI: $14.6-33.0 \%)$ in the study population when considering test sensitivity and specificity. Apparent flock seroprevalence and the odds of $B$. ovis exposure for potential risk factors are presented in Table 2.

Of the 82 flocks sampled for this study, 31\% (25/82) were from the Northwest region, 24\% (20/82) were from the Northeast region, 7\% (6/82) were from the West region, $17 \%(14 / 82)$ were from the South-central region, and $21 \%(17 / 82)$ were from the Southeast region. Flocks in the South-central region had the highest apparent seroprevalence (57\%), and contrary to the animal data, there was a statistically significant difference between seroprevalence in flocks in the five regions $(p=0.009)$ (Table 2) according to the Fisher's exact test.

Of all flocks sampled, $24 \%(20 / 82)$ were very small, $30 \%(24 / 82)$ were small, $39 \%(32 / 82)$ were medium, and $7 \%(6 / 82)$ of flocks were large. An average of two rams per very small flock (95\% CI: 2-3; median: 2 ), seven rams per small flock (95\% CI: 6-8; median: 7), 32 rams per medium flock (95\% CI: 25-38; median: 28 ), and 216 rams per large flock (95\% CI: 115-317; median: 194) were tested. Apparent flock seroprevalence was $10 \%$ in very small flocks, $4 \%$ in small flocks, $31 \%$ in medium flocks, and $83 \%$ in large flocks. There was a statistically significant difference between seroprevalence of flocks of different sizes $(p=0.0002)$ according to the Fisher's exact test, and the odds of a seropositive flock in the medium and large flock sizes was 9.1 and 69 times the odds, respectively, of a seropositive flock in the small flock size category (Table 2).

Seventeen producers disclosed information regarding the ewe-to-ram mating ratio in their flock. Of those 17 flocks, $23.5 \%(4 / 17)$ possessed low ratios (1 to 29 ewes per ram), $53 \%(9 / 17)$ possessed medium ratios (30 to 39 ewes per ram), and 23.5\% (4/17) possessed high ratios ( $\geq 40$ ewes per ram). Flocks with low, medium, and high ewe-to-ram ratios had apparent flock seroprevalence of 25,44 , and $75 \%$, respectively, but this difference was not statistically significant ( $p=0.565)$ according to the Fisher's exact test.

Sixteen producers disclosed information regarding the purchase or introduction of new sheep into their flock. Of those 16 flocks, 56\% (9/16) were classified as open 
Table 2 Apparent flock seroprevalence and odds of B. ovis exposure for risk factors in Wyoming, USA

\begin{tabular}{lllll}
\hline Risk Factor & $N$ & Seroprevalence $^{a}$ & Odds Ratio $(95 \% \mathrm{Cl})$ & $P$ value \\
\hline Region $^{\mathrm{b}}$ & & & & \\
Southeast & 17 & $5.9 \%(1 / 17)$ & Reference & - \\
Northwest & 25 & $12.0 \%(3 / 25)$ & $1.99(0.21$ to 60.81$)$ & 0.575 \\
Northeast & 20 & $25.0 \%(5 / 20)$ & $4.67(0.62$ to 134.15$)$ & 0.294 \\
West & 6 & $16.7 \%(1 / 6)$ & $3.01(0.069$ to 131.62$)$ & 0.575 \\
South-central & 14 & $57.1 \%(8 / 14)$ & $17.34(2.39$ to 502.01$)$ & 0.012 \\
Flock Size & & & & \\
Small & 24 & $4.2 \%(1 / 24)$ & Reference & - \\
Very Small & 20 & $10.0 \%(2 / 20)$ & $2.36(0.18$ to 78.42$)$ & 0.516 \\
Medium & 32 & $31.3 \%(10 / 32)$ & $9.06(1.51$ to 237.89$)$ & 0.018 \\
Large & 6 & $83.3 \%(5 / 6)$ & $69.00(5.81$ to $>999.99)$ & $<0.001$
\end{tabular}

Ewe-to-ram Ratio

$\begin{array}{lllll}\text { Medium } & 9 & 44.4 \%(4 / 9) & \text { Reference } & - \\ \text { Low } & 4 & 25.0 \%(1 / 4) & 0.47(0.01 \text { to } 6.07) & 0.587 \\ \text { High } & 4 & 75.0 \%(3 / 4) & 3.21(0.254 \text { to } 116.89) & 0.587\end{array}$

Purchase of Outside Sheep

$\begin{array}{lllll}\text { No } & 7 & 28.6 \%(2 / 7) & \text { Reference } & - \\ \text { Yes } & 9 & 55.6 \%(5 / 9) & 2.83(0.35 \text { to } 32.03) & 0.340\end{array}$

${ }^{\mathrm{a}}$ Parenthesis indicate proportion of seropositive flocks per category

${ }^{\mathrm{b}}$ Indicates significant difference between seroprevalence by Fisher's exact test at $p<0.05$

flocks, or those that possessed one or more sheep, regardless of gender or age, obtained outside the original flock between 2015 and 2016. The other 44\% (7/16) of flocks were considered closed flocks, or those that did not contain any sheep obtained outside the original flock during that year. Apparent flock seroprevalence of open flocks was $56 \%$ compared to $29 \%$ for closed flocks, and this difference was not statistically significant $(p=0.340)$ according to the Mid-p exact test.

Two variables (region and flock size) were incorporated into a multiple logistic regression model, but the results did not identify any significant factors. One explanation for this result was the relatively large amount of missing data; the model eliminated 25 observations (i.e. flocks) from the analysis due to missing information.

\section{Discussion}

\section{Seroprevalence of $B$. ovis in Wyoming}

In Wyoming, the estimated true animal and flock seroprevalence of B. ovis in the study populations was 0.53 and $22.5 \%$, respectively. Our animal seroprevalence estimate, based on serologic testing, is much lower than prevalence estimates published elsewhere, including $14.9 \%$ in Idaho and Oregon, $27.1 \%$ in Utah, $67.5 \%$ in New Mexico, and $10.0 \%$ in rams across Colorado, Wyoming, and Utah [10, 21-23]. Some of these studies used testicular palpation and bacterial culture to estimate prevalence in rams, and others relied on samples collected from rams suspected of $B$. ovis exposure or samples collected from rams chosen by producers. Thus, these factors may have led to an overestimation of true animal prevalence. Conversely, this study included samples collected from both ewes and clinically normal rams, which may explain our lower animal seroprevalence estimate in the study population.

Reduced animal seroprevalence paired with elevated flock seroprevalence suggests few animals have been exposed to B. ovis, but many flocks contain at least one seropositive animal. This finding coincides with other international studies. Souza et al. [24] reported animal and flock seroprevalence of 0.72 and $8.62 \%$, respectively, while surveying 700 sheep in 58 Brazilian flocks. Sergeant [19] reported $10.8 \%$ of rams were seropositive for B. ovis and $32.9 \%$ of flocks contained at least one seropositive ram in New South Wales. Machado et al. [15] observed $2.89 \%$ of rams and $2.50 \%$ of flocks tested seropositive for B. ovis in Brazil, while Arsenault et al. [25] surveyed 250 rams from 30 Canadian flocks and found no serological evidence of $B$. ovis.

In conclusion, the low number of seropositive sheep in this study $(22 / 2423)$ may suggest $B$. ovis is a negligible disease in Wyoming; however, increased seroprevalence at the flock-level $(18 / 82)$ is concerning and demonstrates the need for further outreach and surveillance.

\section{Significant risk factors}

This study reported statistically significant differences between apparent $B$. ovis seroprevalence in flocks from different regions and different flock sizes. In a similar study, Pinheiro et al. [26] uncovered differences in $B$. ovis seroprevalence when comparing 23 flocks from three regions in Brazil.

Regarding flock size, previous studies reported lower seroprevalence in small flocks compared to medium-sized flocks, and rams from large flocks were 14 times more likely to become infected than rams from small flocks [14, 20]. Our study corroborates these findings; however, one potential pitfall is the presence of confounding flock factors. Carrera Chávez et al. [14] reported significantly higher seroprevalence in rams belonging to flocks with semi-intensive production systems, which were characterized as those that graze sheep on open grassland or rangeland, offer complement feed to increase productivity, have an average flock size of $208 \pm 264$ sheep, and an average ewe-to-ram ratio of $41.3 \pm 16.7$ ewes per ram. Although we did not have a formal production system classification for flocks in this study, large flocks in the U.S. are often associated with regions and management systems that involve grazing sheep on open rangeland, resulting in interactions with other potentially infected sheep [27]. Moreover, some regions in Wyoming have more access to 
public land, which can be used for shared grazing, and this may influence the average flock size in these areas. Disparities in these variables (region and flock size) can influence management strategies, and in turn, influence the likelihood of B. ovis exposure in a flock.

No significant differences in apparent flock seroprevalence and the ewe-to-ram mating ratio were observed. Likewise, Ficapal et al. [20] did not conclude correlation between ewe-to-ram mating ratio of a flock and the bacteriological, serological, or clinical status of B. ovis. In contrast, Carrera Chávez et al. [14] observed highest $B$. ovis seroprevalence in rams from flocks with low eweto-ram mating ratios (16 to 30 ewes per ram), suggesting that $B$. ovis causes reduced fertility in rams and results in a lower capacity to service ewes. If this occurs, producers require additional rams to re-service the open ewes, therefore, decreasing the ewe-to-ram ratio (i.e., more rams needed to breed fewer ewes). One potential explanation for this conflicting result in our study is a lack of sufficient power, given we only had information from 17 flocks. While this study did not identify ewe-toram mating ratio as a significant risk factor, it still may be indicative of $B$. ovis infection, and breeding rams should be tested before re-servicing open ewes to prevent increased disease transmission.

No statistically significant differences were observed in apparent flock seroprevalence between operations that purchased outside sheep (i.e., open flocks) and operations that did not purchase outside sheep (i.e., closed flocks). This finding may also be an artifact of low sample size $(n=16)$. It contrasts with Santos et al. [28], who reported significantly higher $B$. ovis seroprevalence in Brazilian flocks that purchased outside animals compared to those that did not. They also reported the odds of $B$. ovis exposure in open flocks was more than six times the odds of exposure in closed flocks [28]. In our study, the odds of $B$. ovis exposure in open flocks was greater than three times the odds of exposure in closed flocks, but not significant at this level. An increased sample size in the future may corroborate Santos et al.'s finding.

Similar to other studies, statistically significant differences were reported between apparent seroprevalence and age groups. Some authors have concluded probability of infection merely increases with age, while others hypothesize that $B$. ovis seroprevalence correlates with sexual activity $[15,18,20]$. In this study, there were no seropositive lambs, but there was increased apparent seroprevalence in yearling and adult sheep, with the highest seroprevalence in aged sheep. This provides support for the theory that probability of infection increases with age. While the risk of $B$. ovis exposure may be highest in animals with increased sexual activity in controlled settings, highest seroprevalence in older animals in this study is likely due to the increased number of breeding seasons and numerous exposures to $B$. ovis in a natural setting. The results also suggest it may be beneficial to segregate yearling rams from adult and aged rams outside the breeding season to reduce disease spread.

Statistically significant differences between apparent seroprevalence and different breed types were observed. More specifically, the wool breeds (e.g., Merinos and Rambouillets) had lower apparent seroprevalence than other breed types. Similarly, Sergeant [19] observed Merino rams had significantly lower seroprevalence than other British breed rams. In our study, confounding factors may have played a role in seroprevalence differences. Specific breeds are often associated with certain production systems and/or flock sizes, which may influence likelihood of B. ovis infection [14]. A second explanation is due to variation in genetic makeup of different breeds that may influence sexual behaviors, growth rates, and disease susceptibility [20, 29].

No statistically significant differences were observed in apparent animal seroprevalence between regions, gender, or time of sample collection. Few studies have analyzed location of an animal as a risk factor associated with $B$. ovis infection, and to our knowledge, none have reported statistical significance $[15,26,28,30]$. That said, each region of Wyoming contained at least one seropositive animal, emphasizing the importance of proper documentation of disease status before purchasing sheep, regardless of location.

Contrary to our hypothesis, there was no statistically significant difference between apparent seroprevalence in rams and ewes in Wyoming, which reinforces results from other studies [31, 32]. This finding suggests that, when sampled on a larger scale, similar proportions of rams and ewes test seropositive; it also supports the fact that, once introduction occurs, B. ovis spreads to both rams and ewes. Thus, even if breeding rams are tested and seropositive rams culled on an annual basis, $B$. ovis may still be maintained by ewes in the flock.

Lastly, we hypothesized apparent seroprevalence would be significantly higher after the breeding season compared to before the breeding season because $B$. ovis is sexually transmitted during the breeding season $[7,8$, 33]. In contrast, we observed higher apparent seroprevalence before the breeding season than after the breeding season. Explanations for this finding may include: 1) infection and transmission of $B$. ovis is occurring more often directly from ram-to-ram before the breeding season, 2) there is a lack of seroconversion in animals after $B$. ovis exposure, 3) samples were collected outside the optimal ELISA detection window (i.e., 19-36 days after B. ovis exposure) [34], or 4) high correlation of the variables, gender and time of sample collection, relative to the breeding season. The majority of samples collected before the breeding season were from rams and most 
samples collected after the breeding season were from ewes; therefore, we could not draw strong conclusions about seropositivity and time of sample collection relative to the breeding season alone. To our knowledge, no previous studies have considered time of sample collection relative to breeding season as a possible risk factor for seropositivity, but a more comprehensive look into this variable may be useful in the future.

\section{Potential bias}

The initial sampling frame for apparent animal seroprevalence was a list of Wyoming sheep producers obtained from the Wyoming Woolgrower's Association. While this sampling frame may not be truly representative of all sheep in Wyoming, it was our best available resource. We offered free serologic testing to producers in exchange for cooperation in the study, generating bias towards producers suspecting disease or otherwise interested in disease surveillance. Sheep included in the apparent animal seroprevalence estimate were a convenient subset from 18 producer-selected flocks and represented only a small portion of all breeding sheep in Wyoming. Furthermore, information about sheep and flocks were subject to producers' discretion. Additional flocks included in the apparent flock seroprevalence estimate were ones belonging to producers that sent samples to WSVL for diagnostic testing due to suspected disease or routine annual testing. The latter (i.e., routine annual testing) creates bias towards the inclusion of likely seronegative flocks because of the producers' adherence to certain disease management guidelines, while the former (i.e., suspected disease) creates bias towards the inclusion of likely seropositive flocks. We assumed the total number of ram samples sent in for diagnostic testing at WSVL were all breeding rams on the operation, and we used that number to classify flocks into size categories. This could have created miscalculations during our flock size analysis. Diagnostic sensitivity and specificity of the ELISA used was not $100 \%$, resulting in the possibility of false positive and negative results in this study. In a flock of 500 sheep with known B. ovis infection, the ELISA would correctly identify 482 infected sheep and misdiagnose 18 sheep as false negatives. However, in a flock of 500 B. ovis-free sheep, this ELISA would misdiagnose two sheep as false negatives, which is problematic for disease control. For apparent animal and flock seroprevalence, we considered indeterminate results as seronegative, as to not over-estimate the number of seropositive results. Finally, the low number of seropositive results (22 sheep and 18 flocks) combined with missing data limited our interpretation of the multiple logistic regression models.

\section{Conclusions}

This study was the first to estimate true flock seroprevalence of ovine brucellosis in a study population in Wyoming, with $22.5 \%$ of flocks containing at least one seropositive animal. Estimated true animal seroprevalence of $B$. ovis in the study population $(0.53 \%)$ was lower than in other studies conducted in the western U.S. The most important risk factors associated with seroprevalence included age and breed type for animal seroprevalence, and region and flock size for flock seroprevalence. Animal seroprevalence gradually increased from yearling to aged sheep, suggesting older sheep that have undergone numerous breeding seasons are more likely to test seropositive and may continue to infect others due to the chronic nature of this bacterial infection. The separation of young rams from older rams outside the breeding season to limit the spread of $B$. ovis is a possible preventive strategy. Wool breeds had lower animal seroprevalence compared to other breed types, suggesting Rambouillet and Merino breeds are either less susceptible to $B$. ovis infection or additional confounding variables contribute to this difference. Flock seroprevalence was different among regions in Wyoming, demonstrating that flocks in certain areas likely share other characteristics that influence $B$. ovis seroprevalence. Lastly, flock seroprevalence generally increased as flock size increased, which may be attributed to variations in management strategies that depend on overall flock purpose. Other variables did not have a clear influence on seroprevalence of B. ovis in this study; however, maintenance of open flocks and lower ewe-to-ram ratios may be indicative of $B$. ovis infection in a flock.

\section{Methods}

The University of Wyoming Institutional Animal Care and Use Committee (IACUC) approved the study and sampling method (protocol \#200150622KS00178-01).

\section{Study population and sample collection}

The study area was the state of Wyoming, USA, which is approximately $253,819 \mathrm{~km}^{2}$ in size with suitable land for sheep production ranging from 900 to more than 3, $000 \mathrm{~m}$ in elevation. Figure 1 shows the study area and approximate locations of sampled operations for estimated B. ovis flock seroprevalence) [13]. The state had 293 sheep operations in 2015 and ranked third in the nation for the largest breeding sheep population with 210,000 ewes and 7,000 rams $[35,36]$. Five geographic regions were created based on agricultural districts used by the United States Department of Agriculture (USDA) and the National Agricultural Statistics Services (NASS).

Though the total number of sheep operations in Wyoming was known, contact information was not available. Hence, the starting sampling frame was a list of 82 Wyoming sheep producers obtained from the Wyoming 


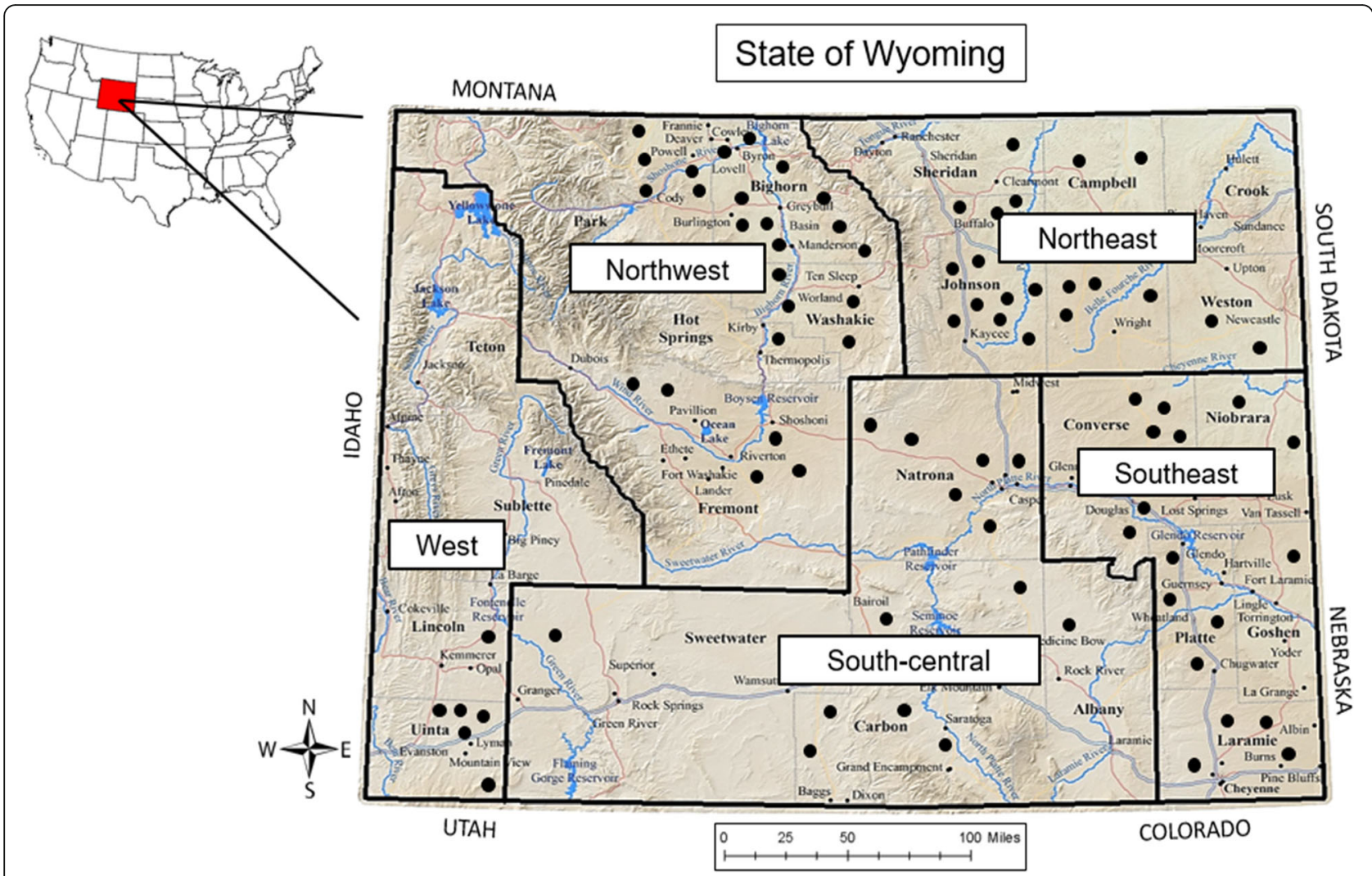

Fig. 1 Study area and approximate locations of sampled operations for estimated B. ovis flock seroprevalence

Woolgrower's Association [37]. Producers were contacted by telephone, email, or brochures sent in the mail and were offered free serological testing for a portion of their flock in exchange for cooperation in the study. Eighteen producers expressed interest, and a questionnaire approved by the University of Wyoming's Institutional Review Board (IRB) was utilized to obtain additional information about flock size, predominant breeds, and breeding practices. Refer to "2015-2016 Sheep Brucellosis Survey" in the Additional file 1 for a full list of questions.

Permission to enter property and collect blood samples was obtained from the 18 operations prior to sample collection, and sampling dates were chosen based on accessibility of sheep between August 2015 and May 2016. Sheep included in the animal seroprevalence estimate were a convenient subset of available ewes and, if possible, all breeding and yearling rams from 18 producer-selected operations in Wyoming. Yearling rams and ram lambs that had not undergone breeding, but were housed with mature, breeding rams were included in the initial study population. If rams were not available for sampling at the time of blood collection (e.g., sampling occurred during pregnancy checking of ewes), results from ram samples submitted to the Diagnostic Serology Lab at the Wyoming State Veterinary Laboratory (WSVL) by the same producer between January 2015 and May 2017 were used. If ewes were not available for sampling at time of blood collection (e.g., sampling occurred during BSEs of rams), results from ewe samples submitted to WSVL by the same producer were used.

During sample collection, a properly trained student, producer, veterinarian, or veterinary technician drew 5$10 \mathrm{~mL}$ of blood via jugular venipuncture of physically healthy sheep using $10 \mathrm{~mL}$ blood collection tubes (BD Vacutainer, Franklin Lakes, NJ, USA) and needle holders (BD Vacutainer, Franklin Lakes, NJ, USA) with disposable $1 \frac{1}{2}$ inch, 18-20 gauge needles (Greiner Bio-One, Kremsmünster, Austria). Relevant information was recorded for each sheep (i.e., location, gender, age, breed, and date of sample collection) at the time of blood collection. Blood was allowed to clot before transportation back to WSVL on ice. Sera was separated from blood samples using a centrifuge, decanted into labeled tubes, and stored at $-20^{\circ} \mathrm{C}$ until serologic testing.

The sample size required for each operation was calculated post-collection after questionnaires revealed the total number of breeding rams and ewes in the flock [38]. For operations in which breeding ewes and rams were available for sampling $(n=9)$, we used the total number of breeding sheep for population size, expected true seroprevalence of $10 \%$ [10], acceptable error of $5 \%$, 
confidence level of $95 \%$, and test sensitivity and specificity of 96.3 and $99.6 \%$ [39], respectively. For operations in which only rams were available for sampling $(n=9)$, we used the total number of breeding rams in the flock for population size and the same inputs above. In some operations, the number of samples collected was not equivalent to the required sample size because samples were collected prior to the sample size calculations. A total of 2423 sheep were used to estimate true seroprevalence in the study population, as shown in Table 3.

\section{Additional flock inclusion criteria}

To bolster confidence in the flock seroprevalence estimate in the study population, we used the samples from the 18 producer-selected operations from above, in conjunction with samples that other Wyoming producers submitted to the Diagnostic Serology Lab at the WSVL for B. ovis testing between January 2015 and May 2017. These additional flocks were not used in the animal seroprevalence estimation and risk factor analysis because the questionnaire was not distributed to these producers. Additional flocks eligible for inclusion in the flock seroprevalence estimation required a WSVL submission form indicating producer surname, producer and/or veterinary clinic address within Wyoming, and a minimum of two samples from different sheep in the same flock. The flock was excluded if there was no producer surname or if the surname matched one of the 18 producer-selected operations already included. Some forms lacked a producer address; however, if the veterinary clinic listed on the form was located within the state, an assumption was made that the flock resided in the same region. A minimum requirement of two samples on each submission form allowed the inclusion of small flocks whose main purpose is club lamb or 4-H lamb production. Some producers submitted multiple forms for diagnostic testing for their flocks, and to reduce confounding, only one form per producer surname was considered. If a single producer submitted multiple forms, the submission form with the most samples was used to achieve a more accurate estimation of overall flock size. If a single producer submitted multiple forms containing the same number of samples, the form that was submitted first was used.

The sample size required for the flock seroprevalence estimate was 173 when using a total population size of 293 , expected true seroprevalence of $50 \%$, acceptable error of $5 \%$, confidence level of $95 \%$, and test sensitivity and specificity of 96.3 and 99.6\% [39], respectively. That said, we had access to sera from 82 Wyoming flocks (18 producer-selected flocks and 64 flocks from WSVL submission forms), which we used to estimate true flock seroprevalence in the study population.

Table 3 Total blood samples collected from 2423 sheep in 18 flocks in Wyoming, USA

\begin{tabular}{|c|c|c|c|c|c|c|}
\hline \multirow[t]{2}{*}{ Flock } & \multicolumn{2}{|c|}{ Total number of sheep } & \multicolumn{2}{|c|}{ Number of blood samples } & \multirow{2}{*}{$\begin{array}{l}\text { Required } \\
\text { sample } \\
\text { size }\end{array}$} & \multirow{2}{*}{$\begin{array}{l}\text { Total } \\
\text { samples }\end{array}$} \\
\hline & Rams & Ewes & Rams & Ewes & & \\
\hline 1 & 50 & 1600 & 23 & N/A & 38 & 23 \\
\hline 2 & 75 & 2300 & 50 & 196 & 142 & 246 \\
\hline 3 & 10 & 35 & 10 & N/A & 10 & 10 \\
\hline 4 & 210 & 6000 & 185 & 157 & 148 & 342 \\
\hline 5 & 15 & 120 & 9 & 16 & 72 & 25 \\
\hline 6 & 40 & 1000 & 40 & N/A & 32 & 40 \\
\hline 7 & 105 & 2500 & 90 & 144 & 143 & 234 \\
\hline 8 & 12 & 400 & 9 & N/A & 12 & 9 \\
\hline 9 & 400 & 8000 & 393 & 64 & 149 & 457 \\
\hline 10 & 100 & 2000 & 66 & N/A & 61 & 66 \\
\hline 11 & 202 & 6000 & 202 & N/A & 87 & 202 \\
\hline 12 & 55 & 2100 & 55 & N/A & 41 & 55 \\
\hline 13 & 25 & 80 & 15 & 20 & 62 & 35 \\
\hline 14 & 70 & 730 & 67 & 69 & 128 & 136 \\
\hline 15 & 35 & 950 & 32 & 92 & 135 & 124 \\
\hline 16 & 250 & 9500 & 187 & 188 & 149 & 375 \\
\hline 17 & 50 & 1250 & 35 & N/A & 38 & 35 \\
\hline 18 & NA & NA & 9 & N/A & NA & 9 \\
\hline Total & & & 1477 & 946 & 1447 & 2423 \\
\hline
\end{tabular}

$\mathrm{N} / \mathrm{A}$ indicates information and/or samples were not available 


\section{Classification and description of risk factors}

Continuous and categorical information obtained at the time of sample collection, via questionnaire, or WSVL submission forms was utilized to create categorical risk factors for animals and flocks. Possible risk factors evaluated for animals included region, gender, age group, primary breed type, and time of sample collection relative to the breeding season. Possible risk factors evaluated for flocks included region, flock size, ewe-to-ram mating ratio, and the introduction of new sheep into the flock.

For regional classification, both animals and flocks were classified into one of five categories based on the county where breeding occurred for the 18 producer-selected operations, and the location of producer and/or veterinary clinic address for the 64 operations that sent samples to WSVL for B. ovis testing. Sheep were classified into one of two gender categories. Sheep were classified into one of four categories based on known age (i.e., ear tag or producer knowledge) or estimated age (i.e., number or condition of teeth). Age categories include lambs ( $<1$ year of age), yearlings ( $\geq 1$ and $<2$ years of age), adults ( $\geq 2$ and $<6$ years of age), and aged sheep ( $\geq 6$ years of age), which was similar to categorization in Van Metre et al. [10] and Wiemer and Ruttle [23]. Sheep were classified into one of three categories based on the primary purpose of the breed or crossbreed. Sheep breeds can be dual purpose, but most excel in the production of wool, meat, or dairy. There were no dairy breeds in this study; therefore, categories included wool breeds, meat breeds, and multi-purpose breeds. Wool breeds include Rambouillet and Merino purebreds or crossbreeds. Meat breeds include Dorset, Hampshire, Suffolk, and Texel breeds or crossbreeds. Multi-purpose breeds include Columbia, Finn sheep, and Targhee breeds or crossbreeds. Sheep were classified into one of two categories based on date of sample collection relative to known breeding months, and include sheep sampled before the breeding season and sheep sampled after the breeding season.

Flocks were classified into one of four flock size categories based on the known number of rams in the flock for the 18 producer-selected operations, and the number of ram samples tested at WSVL for the 64 additional operations. Breeding soundness exams and serological testing for B. ovis infection typically occur once per year prior to the breeding season. Therefore, an assumption made was that the total number of ram samples submitted to WSVL for testing was equivalent to the total number of breeding rams in the flock at the given time. Flock size categories included very small (2-3 breeding rams), small (4-10 breeding rams), medium (11-100 breeding rams), and large (> 100 breeding rams). Flocks were classified into one of three categories based on the number of ewes an average ram can breed in one season. This number depends on multiple aspects, including the age and breeding experience of the ram, the environment in which the ram is working, and the number of ewes in the flock. We based our categories on recommendations from producers and categories from similar studies. Flock categories for ewe-to-ram mating ratios included low (1 to 29 ewes per ram), medium (30 to 39 ewes per ram), and high ( $\geq 40$ ewes per ram). Flocks were classified into one of two categories based on whether the producer purchased and introduced new sheep into the flock that year (i.e., open flock) or not (i.e., closed flock).

\section{Serologic testing and quality control}

Serologic testing was conducted at WSVL using the National Veterinary Services Laboratory (NVSL) B. ovis ELISA. Currently, this is the only ELISA licensed in the U.S. for detection of B. ovis antibodies in sheep and goat serum, and diagnostic sensitivity and specificity is reported to be 96.3 and $99.6 \%$, respectively [39]. The antigen used for the assay is a hot-saline water soluble extract of the REO 198 strain prepared according to the World Organization for Animal Health recommendations by the Animal and Plant Health Inspection Service and the NVSL (APHIS-NVSL, Ames, IA, USA); controls include "high positive" sera, "low positive" sera, and "negative" sera [1]. NVSL personnel tested the antigen and control sera for potency and sterility, and all antigen and controls used in this study were from the same lot number.

At WSVL, the same two persons prepared and performed all assays. Sample sera were run in duplicate and control sera in triplicate with additional "low positive" and "negative" controls on the back of the plate to ensure consistent development during the assay. During plate preparation, polystyrene micro-titer plates (Nunc A/S, Roskilde, Denmark) were coated with an antigen dilution in carbonate buffer, incubated, and blocked with bovine serum albumin solution (KPL, Inc., Gaithersburg, MD, USA). Prior to running the assay, conjugated Protein G-Biotin from Streptococcus spp. (Sigma-Aldrich Corporation, St. Louis, MO, USA), Vectastain conjugate (Vector Laboratories, Burlingame, CA, USA), Blue Phosphatase substrate (KPL, Inc., Gaithersburg, MD, USA), and APstop solution (KPL, Inc., Gaithersburg, MD, USA) were prepared according to the manufacturers' instructions. During assay procedure, sera, conjugated Protein G-Biotin, and Vectastain conjugate were incubated at $37^{\circ} \mathrm{C}$ in a humidified chamber inside a digital display incubator (Boekel Industries, Inc., Feasterville, Trevose, PA, USA) for $45 \mathrm{~min}, 35 \mathrm{~min}$, and $25 \mathrm{~min}$, respectively. After each incubation, the plate was washed with a high salt wash solution, excess liquid emptied in the sink, and the plate tapped onto absorbent material to remove any non-specifically bound proteins or antibodies. The Blue Phosphatase substrate incubation step was performed at room temperature on a microplate 
shaker (Lab-line Instruments, Inc., Melrose Park, IL, USA), and color development stopped when the low positive control average reached an optical density (OD) between $0.350-0.500$ at a wavelength of $620 \mathrm{~nm}$ on a plate reader (TECAN, Männedorf, Züich, Switzerland; BioTek Instruments, Winooski, VT, USA). The average OD for each sample and control was calculated, and the average sample to positive $(\mathrm{S} / \mathrm{P})$ ratio used to determine sample status. An $\mathrm{S} / \mathrm{P}$ ratio of greater than 0.75 was positive, greater than or equal to 0.40 and less than or equal to 0.75 was indeterminate, and less than 0.40 was negative.

If the average OD for control sera on the front and back of the plate was outside the expected acceptable range of values for that particular lot, the plate was considered invalid and each sample re-tested. If control sera yielded ODs with a difference of greater than $20 \%$, the well with the largest variance was excluded, the percent difference recalculated, and if acceptable (i.e., less than $20 \%$ ), the ODs were then used to calculate an average for the control. If sample sera yielded two ODs with a difference of greater than $20 \%$, the sample was re-tested. When sample sera yielded an indeterminate or positive result, the sample was re-tested to ensure minimal reporting of false positive or false indeterminate results. If a re-tested indeterminate or positive sample had the same result, the average of the S/P ratios was calculated. If a re-tested indeterminate or positive sample had a different result, the sample was re-tested a third time, and the average of the two S/P ratios with the least amount of variance was calculated.

\section{Statistical analysis}

Sheep with seropositive test results were considered positive for $B$. ovis antibodies, and sheep with indeterminate and negative test results were negative for $B$. ovis antibodies. Flocks were considered positive if one or more sheep within the flock had a seropositive result. The apparent animal and flock seroprevalence was calculated, and the Rogan and Gladen (1978) formula was used to estimate true seroprevalence in the animal and flock populations using a sensitivity of $96.3 \%$ and specificity of $99.6 \%[39,40]$ :

$$
\text { True prevalence }=\frac{[\text { Apparent prevalence }+(\text { Specificity-1 })]}{[\text { Sensitivity }+(\text { Specificity }-1)]}
$$

For apparent seroprevalence, we calculated 95\% confidence intervals (CI) using Wilson-Score intervals. For estimated true seroprevalence in the study populations, we calculated 95\% CIs using Blaker's intervals, as recommended by the authors of EpiTools epidemiological calculators provided by AusVet online [38, 41].
For variables that had two levels within the category (i.e. gender, time of sample collection, and purchase of outside sheep), we used the Mid-p exact test with odds ratios and 95\% CIs to compare overall apparent seroprevalence. For variables that had more than two levels within the category (i.e. region, age category, breed type, flock size, and ewe-to-ram ratio), we used the Fisher's exact test to compare overall apparent seroprevalence followed by the Mid-p exact test with odds ratios and 95\% CIs to further compare seroprevalence between levels within each variable. Individual sheep or flocks were excluded from calculations if they lacked the necessary information (e.g., age, breed, ewe-to-ram ratio, etc.) needed for the risk factor analyses. For example, if one sheep had information regarding its gender and breed, but lacked information regarding its age, we included that sheep in the gender and breed analysis and excluded it from the age analysis. Values of $p<0.05$ were considered statistically significant, and only significant variables were incorporated into a multiple logistic regression model. For variables with multiple levels, $p$ values have been adjusted using the false discovery rate (FDR) method. The statistical analyses were performed using RStudio 1.0.136 [42] within $\mathrm{R}$ version 3.4.3.

\section{Additional file}

\section{Additional file 1: 2015-2016 Sheep Brucellosis Survey. (DOCX 589 kb)}

\section{Abbreviations}

B. sheep: Brucella ovis; BSE: Breeding soundness exam; Cl: Confidence interval; ELISA: Enzyme-linked immunosorbent assay; IACUC: Institutional Animal Care and Use Committee; IRB: Institutional Review Board; WET: National

Agricultural Statistics Service; NVSL: National Veterinary Services Laboratory; FROM: Optical density; S/P ratio: Sample to positive ratio; USDA: United

States Department of Agriculture; WSVL: Wyoming State Veterinary Laboratory

\section{Acknowledgements}

The authors thank all Wyoming sheep producers, assistants, and veterinarians that participated in this study. They also acknowledge the many undergraduate students, faculty and staff members of WSVL for their assay expertise and assistance.

\section{Authors' contributions}

The study idea was conceived by KS and ME. KS secured funding, directed laboratory performance, and evaluated ELISA results. ME collected blood samples, administered questionnaires, performed serologic testing, and wrote the manuscript. ME, KS, and BS participated in study design and statistical analysis. ME, KS, BS, and DP provided manuscript revisions. ME, KS, $\mathrm{BS}, \mathrm{TC}$, and DP contributed to the conception and overall design of the study. All authors read and approved the final manuscript.

\section{Funding}

This work was supported by a Wyoming State Agriculture Producer Research Grant and Wyoming Agricultural Experiment Station funding provided through the USDA National Institute of Food and Agriculture, Hatch project \#1008761. This funding agency did not have a role in the study design, sample collection, data analysis, data interpretation, nor manuscript preparation. 


\section{Availability of data and materials}

The datasets generated and/or analyzed during the current study are not publicly available due to cooperating producer privacy and confidentiality, but are available from the corresponding author on reasonable request.

\section{Ethics approval and consent to participate}

The University of Wyoming Institutional Animal Care and Use Committee (IACUC) approved the study and sampling method (protocol \#200150622KS00178-01). Permission to enter property and collect blood samples was obtained from the 18 operations prior to sample collection, and sampling dates were chosen based on accessibility of sheep between August 2015 and May 2016. The University of Wyoming's Institutional Review Board (IRB) approved the "2015-2016 Sheep Brucellosis Survey" questionnaire. Following blood sample collection, the survey was mailed to the 18 participating operations. While the survey was voluntary, consent to the survey was implied if the completed survey was returned to us.

\section{Consent for publication}

Not applicable.

\section{Competing interests}

The authors declare that they have no competing interests.

\section{Author details}

'Department of Veterinary Sciences, University of Wyoming, 1174 Snowy Range Road, Laramie, WY 82070, USA. ${ }^{2}$ Northern Plains Climate Hub, United States Department of Agriculture, Agricultural Research Service, 1701 Center Avenue, Fort Collins, CO 80526, USA.

\section{Received: 24 March 2019 Accepted: 4 July 2019}

\section{Published online: 15 July 2019}

\section{References}

1. World Organization for Animal Health. OIE terrestrial manual 2015. In: Ovine epididymitis (Brucella ovis); 2015. p. 1-13. Available from: http://www.oie. int/fileadmin/Home/eng/Health_standards/tahm/3.07.07_OVINE_EPID.pdf. Cited 26 July 2017.

2. Buddle MB, Boyles BW. A Brucella mutant causing genital disease of sheep in New Zealand. Aust Vet J. 1953;29(6):145-53.

3. Simmons GC, Hall WTK. Epididymitis of rams. Aust Vet J. 1953;29(2):33-40.

4. McGowan B, Shultz G. Epididymitis of rams: clinical description and field aspects. Cornell Vet. 1956;46:277-81.

5. Blasco JM. Brucella ovis infection. In: Infectious and parasitic diseases of livestock. Paris: Editions Tec \& Doc; 2010.

6. Blasco JM. Brucella ovis. In: Animal brucellosis; 1990. p. 351-74. Available from: http://books.google.com/books?hl=en\&lr=\&id=QQFDHBpNnTQC\&oi= fnd\&pg=PA351\&dq=info:GaUXFDBGZPMJ:scholar.google.com\&ots= WmFwnbTD5x\&sig=bTZ9QJ3C21LXmNOuzMhHCXNxuaw.

7. Buddle MB. Observations on the transmission of Brucella infection in sheep. N Z Vet J. 1955;3(1):10-9

8. Hartley WJ, Jebson JL, McFarlane D. Some observations on natural transmission of ovine brucellosis. N Z Vet J. 1955;3(1):5-10.

9. Marco J, Gonzalez L, Cuervo LA, Beltran de Heredia F, Barberan M, Marin C, et al. Brucella ovis infection in two flocks of sheep. Vet Rec. 1994;135:254-6.

10. Van Metre DC, Rao S, Kimberling CV, Morley PS. Factors associated with failure in breeding soundness examination of Western USA rams. Prev Vet Med. 2012;105(1-2):118-26.

11. Estein SM, Fiorentino MA, Paolicchi FA, Clausse M, Manazza J, Cassataro J, et al. The polymeric antigen BLSOmp31 confers protection against Brucella ovis infection in rams. Vaccine 2009; 27 (48): 6704-11.

12. Ridler AL, West DM. Control of Brucella ovis infection in sheep. Vet Clin N Am Food Anim Pract. 2011;27(1):61-6

13. Gardiner L, Rashford B, Hewlett J, Alexander B. State of Wyoming sheep industry: University of Wyoming Extension; 2012. p. 1-22. Available from: http://www.uwagec.org/farmmgt/PUBS/B1229.pdf

14. Chávez JM career, Echavarría Cháirez FG, Aréchiga Flores CF, Bañuelos Valenzuela $\mathrm{R}$, Tórtora Pérez JL. Possible risk factors for serological prevalence of Brucella ovis in Zacatecas, Mexico. Rev Mex Cienc Pecu. 2013;4(1):61-74.

15. Machado G, Santos DV, Kohek I, Stein MC, Hein HE, Poeta AS, et al. Seroprevalence of Brucella ovis in rams and associated flock level risk factors in the state of Rio Grande do Sul , Brazil. Prev Vet Med. 2015;121(12):183-7.

16. Marín CM, Jimenez de Bagues MP, Blasco JM, Gamazo C, Moriyon I, Diaz R. Comparison of three serological tests for Brucella ovis infection of rams using different antigenic extracts. Vet Rec. 1989;125(20):504-8.

17. Ridler A. An overview of Brucella ovis infection in New Zealand. N Z Vet J. 2002;50(sup3):96-8.

18. Robles CA, Uzal FA, Olaechea FV, Low C. Epidemiological observations in a Corriedale flock affected by Brucella ovis. Vet Res Commun. 1998;22(7):43543.

19. Sergeant ESG. Seroprevalence of Brucella ovis infection in commercial ram flocks in the Tamworth area. N Z Vet J. 1994;42(3):97-100.

20. Ficapal A, Jordana J, Blasco JM, Moriyón I. Diagnosis and epidemiology of Brucella ovis infection in rams. Small Rumin Res. 1998;29(1):13-9.

21. Bagley, Paskett ME, Matthews NJ, Stenquist NJ. Prevalence and causes of ram epididymitis in Utah. J Am Vet Med Assoc. 1985;186(8):798-801.

22. DeLong WJ, Waldhalm DG, Hall RF. Bacterial isolated associated with epididymitis in rams from Idaho and eastern Oregon flocks. Am J Vet Res. 1979:40(1):101-2.

23. Wiemer KE, Ruttle JL. Semen characteristics, scrotal circumference and bacterial isolates of fine wool range rams. Theriogenology. 1987;28(5):62537

24. Souza TS, Costa JN, Martinez PM, de Lima CCV, Araújo BR, Costa N, et al. Seroepidemiological survey for Brucella ovis infection in sheep flocks of semi-arid region in Bahia State, Brazil. Vet Zootec. 2011;18(4, Supl. 3):697700.

25. Arsenault J, Girard C, Dubreuil P, Bélanger D. Lack of evidence of Brucella ovis infection in rams infection in rams in Quebec. Can Vet J. 2004;45(4):312.

26. Pinheiro JW, Oliveira AA d F, Mota RA, Agottani JV, de Jesus EM, de Assis ST, et al. Occurance of anti-Brucella ovis antibodies in sheep in the state of Alagoas, Brazil. Vet Zootec. 2009;16(3):500-8.

27. United States Department of Agriculture, Animal and Plant Health Inspection Service, Veterinary Services, National Animal Health Monitoring System. Part I: Reference of sheep management practices in the United States, 2011. Fort Collin; 2012. p. 1-131. Available from: https://www.aphis. usda.gov/animal_health/nahms/sheep/downloads/sheep11/Sheep11_dr Partl.pdf. Cited 24 Jul 2017

28. Santos FA, Higino SS, Azevedo SS, Costa DF, Farias AE, Alves FA, et al. Epidemiological characterization and risk factors associated with Brucella ovis infection in sheep in the Brazilian semiarid. Pesqui Vet Bras. 2013;33(4): 459-63.

29. Marín CM, Jimenez de Bagues MP, Blasco JM, Gabina D. Incidence of reproductive abnormalities in different breeds of rams. Zaragoza: US-Spain Joint Seminar on Sheep Breeding; 1986. p. 303-7.

30. Clementino IJ, Alves CJ, de Azevedo SS, Paulin LM, Medeiros KA. Seroepidemiological survey and risk factors for Brucella ovis infection in rams of the semiarid region of Paraiba, northeastern Brazil. Pesqui Vet Bras. 2014; 27(4):137-43.

31. Araújo BR, Leite MDX, Souza TS, Lima CCV, Costa JN, Lima EB, et al. Seroepidemiology of sheep brucellosis in the microregion of Feira de Santana, BA, Brazil. Braz J Vet Res Anim Sci. 2013;50(2):129-35.

32. Silva N dos $S$, de Barros IN, Dasso MG, de Almeida M das GÁR, Laborda S da S, Annunciation AVM, et al. Detection of Brucella ovis antibodies in Bahia. 2009; Available from: http://www.repositorio.ufba.br:8080/ri/handle/ri/5384. Cited 7 Jul 2017.

33. Brown GM, Pietz DE, Price DA. Studies on the transmission of Brucella ovis infection in rams. Cornell Vet. 1973;63(1):29-40.

34. Ridler A, Smith S, West D. Seroconversion and semen shedding in rams experimentally infected with Brucella ovis. N Z Vet J. 2014;62(1):47-50.

35. Brandt R, Hussey N. Wyoming agricultural statistics report 2015. Cheyenne: USDA, NASS, Wyoming Field Office; 2015. p. 1-91.

36. National Agricultural Statistics Service. 2012 census of agriculture- state data 2012. Available from: https://www.nass.usda.gov/Publications/AgCensus/2 012/Full_Report/Nolume_1,_Chapter_2_US_State_Level/st99_2_044_044.pdf. Cited 18 Dec 2018.

37. Wyoming Woolgrowers Auxiliary. Wyoming sheep breeders directory. 2014.

38. Sergeant E. EpiTools epidemiological calculators: Ausvet Pty Ltd; 2017 Available from: http://epitools.ausvet.com.au. Cited 2 Aug 2017

39. Gall D, Nielsen K, Vigliocco A, Smith P, Perez B, Rojas X, et al. Evaluation of an indirect enzyme-linked immunoassay for presumptive serodiagnosis of Brucella ovis in sheep. Small Rumin Res. 2003;48(3):173-9. 
40. Rogan WJ, Gladen B. Estimating prevalence from the results of a screening test. Am J Epidemiol. 1978;107(1):71-6.

41. Reiczigel J, Földi J, Ozsvári L. Exact confidence limits for prevalence of a disease with an imperfect diagnostic test. Epidemiol Infect. 2010;138(11): 1674-8.

42. RStudio Team. RStudio. Boston: RStudio; 2016. Available from: http://www. rstudio.com/.

\section{Publisher's Note}

Springer Nature remains neutral with regard to jurisdictional claims in published maps and institutional affiliations.

- fast, convenient online submission

- thorough peer review by experienced researchers in your field

- rapid publication on acceptance

- support for research data, including large and complex data types

- gold Open Access which fosters wider collaboration and increased citations

- maximum visibility for your research: over $100 \mathrm{M}$ website views per year

At $\mathrm{BMC}$, research is always in progress. 